\title{
A Risk Signature with Autophagy-Related Long Noncoding RNAs for Predicting the Prognosis of Clear Cell Renal Cell Carcinoma: Based on the TCGA Database and Bioinformatics
}

\author{
Yundong Xuan, ${ }^{1,2}$ Weihao Chen, ${ }^{2}$ Kan Liu, ${ }^{2}$ Yu Gao, ${ }^{2}$ Shidong Zuo, ${ }^{1,2}$ Baojun Wang $\mathbb{D},{ }^{2}$ \\ Xin $\mathrm{Ma}\left(\mathbb{D},{ }^{2}\right.$ and $\mathrm{Xu}$ Zhang $\mathbb{D}^{2}$ \\ ${ }^{1}$ Medical School of Chinese PLA, Beijing 100853, China \\ ${ }^{2}$ Department of Urology, The Third Medical Centre, Chinese PLA (People's Liberation Army) General Hospital, \\ Beijing 100853, China
}

Correspondence should be addressed to Baojun Wang; baojun40009@126.com, Xin Ma; urologist@foxmail.com, and Xu Zhang; xzhang@foxmail.com

Received 26 August 2020; Revised 2 April 2021; Accepted 23 April 2021; Published 7 May 2021

Academic Editor: Mingjun Shi

Copyright ( 2021 Yundong Xuan et al. This is an open access article distributed under the Creative Commons Attribution License, which permits unrestricted use, distribution, and reproduction in any medium, provided the original work is properly cited.

Background. Disorders of autophagic processes have been reported to affect the survival outcome of clear cell renal cell carcinoma (ccRCC) patients. The purpose of our study was to identify and validate the candidate prognostic long noncoding RNA signature of autophagy. Methods. Transcriptome profiles were obtained from The Cancer Genome Atlas. The autophagy gene list was obtained from the Human Autophagy Database. Based on coexpression analysis, we obtained a list of autophagy-related lncRNAs (ARlncRNAs). GO enrichment analysis and KEGG pathway analysis were conducted to explore the functional annotation of these ARlncRNAs. Univariate and multivariate Cox regression analyses were conducted to elucidate the correlation between overall survival and the expression level of each ARlncRNAs. We then established a prognostic signature that was a linear combination of the regression coefficients from the multivariate Cox regression model $(\beta)$ multiplied by the expression levels of the respective ARlncRNAs in the training cohort. The predictive performance was tested in the validation cohort. Additionally, the independence of the risk signature was assessed, and the relationship between the risk signature and conventional clinicopathological features was explored. Results. Seven autophagy-related lncRNAs with prognostic value (SNHG3, SNHG17, MELTF-AS1, HOTAIRM1, EPB41L4A-DT, AP003352.1, and AC145423.2) were identified and integrated into a risk signature, dividing patients into low-risk and high-risk groups. The risk signature was independent of conventional clinical characteristics as a prognostic indicator of ccRCC (HR, 1.074, 95\% confidence interval: 1.036-1.113, $p<0.001$ ) and was valuable in the prediction of ccRCC progression. Conclusion. Our risk signature has potential prognostic value in ccRCC, and these ARlncRNAs may play a significant role in ccRCC tumor biology.

\section{Introduction}

Renal cell carcinoma (RCC), a principal malignancy of the renal tubular epithelium, ranks third among urinary cancers 1 [1]. Characterized by multifarious genetic features [2], RCC is composed of different histopathologic subtypes, of which clear cell renal cell carcinoma (ccRCC) represents the principle pathologic subtype, accounting for $70 \%$ to $80 \%$ of RCCs
[3]. The prognosis of ccRCC varies between patients with complicated genetic mutations, such as von Hippel-Lindau (VHL), PBRM1, and BAP1 [4]. Surgery remains the principal treatment for ccRCC due to its therapeutic value, but $40 \%$ of ccRCC patients will eventually suffer distant metastasis [5], and few exhibit a positive response to radiotherapy or chemotherapy [6]. Neither immunologic nor targeted therapy can definitely benefit ccRCC patients [7]. Therefore, 
predicting the progression and prognosis of ccRCC by seeking novel effective biomarkers might facilitate the therapeutic schedule and the evaluation of survival status.

Autophagy, a process in which cells engulf proteins and organelles by forming double-membraned autophagic vesicles where degradation occurs, is regarded as a recycling of organelles and an adaptation of metabolism. Autophagy plays a tumor-suppressor role by inhibiting the selection and expansion of tumor-initiating cells early in tumor development [8]. However, increasing evidence in established tumors suggests that autophagy can help cope with environmental or intracellular stresses, such as hypoxia, nutrient shortage, or cancer therapy, thereby promoting tumor growth [9-11]. Although an increasing number of studies have sought to identify novel potential targets by probing the autophagy pathway, and autophagic drugs have been reported to induce renal cancer cell death, the mechanism is still to be clarified [12].

Long noncoding RNAs (lncRNAs), a variety of noncoding RNA, participate in many cellular processes with multitudinous functions by modulating gene expression at the epigenetic, transcriptional, and posttranscriptional levels [13-15]. Accumulating evidence suggests that lncRNAs can target autophagy-related genes at both the transcriptional and posttranscriptional levels to regulate the autophagy pathway, and lncRNAs regulate various proteins that function in the autophagy process $[16,17]$. Dysregulated lncRNAs are involved in ccRCC progression and dissemination [18]. It has been suggested that autophagy-related lncRNAs can exert their action in tumor regulation [19].

The potential value of autophagy-related lncRNAs in evaluating the prognosis of ccRCC patients and their role as potential therapeutic targets have yet to be fully explored. Here, we sought to identify an autophagy-related lncRNA signature in ccRCC and to advance more personalized treatment guidelines for ccRCC through bioinformatic analysis.

\section{Materials and Methods}

2.1. Data Acquisition Processing. In our study, RNA-seq transcriptome data of which the workflow type was HTSeqFPKM and clinical information data were downloaded from the TCGA database (https://portal.gdc.cancer.gov/) [20]. The lncRNA matrix and mRNA matrix were extracted from transcriptome profiling, respectively, by gene annotation. A list consisting of 232 autophagy genes was obtained from the Human Autophagy Database (HADb, http://autophagy.lu/ clustering/index.html) (Table S1). Then, the expression matrix of 210 autophagy genes was extracted combing the mRNA matrix with autophagy genes list (Table S2), and 21 genes were abandoned due to lacking expressing information. In addition, the clinicopathological features included survival status, survival time, age, sex, International Society of Urological Pathology (ISUP) grade, and American Joint Committee on Cancer (AJCC) stage.

2.2. Autophagy-Related IncRNAs Screening. The Pearson correlation test was performed to screen autophagy-related lncRNAs based on 210 autophagy genes and all lncRNAs as
TABLE 1: Clinical characteristics of ccRCC patients in the TCGA database.

\begin{tabular}{lccc}
\hline Characteristics & & Total & $\%$ \\
\hline Age at diagnosis $(\mathrm{y})$ & & $58(26 \sim 90)$ & \\
Gender & Female & 191 & 35.57 \\
& Male & 346 & 64.43 \\
Stage & I & 269 & 50.37 \\
& II & 57 & 10.67 \\
& III & 125 & 23.41 \\
Grade & IV & 83 & 15.55 \\
& G1 & 14 & 2.65 \\
& G2 & 230 & 43.48 \\
T stage & G3 & 207 & 39.13 \\
& G4 & 78 & 14.74 \\
& T1 & 275 & 51.21 \\
& T2 & 69 & 12.85 \\
N stage & T3 & 182 & 33.89 \\
& T4 & 11 & 2.05 \\
M stage & N0 & 240 & 93.39 \\
& N1 & 17 & 6.61 \\
& M0 & 426 & 84.36 \\
& M1 & 79 & 15.64 \\
\hline \multirow{4}{*}{ S1 } & & &
\end{tabular}

mentioned. A lncRNA with a correlation coefficient $|R|>$ 0.3 and $p<0.001$ was considered an autophagy-related lncRNA. The "limma" package in R software was then used to screen [21] differentially expressed autophagy-related lncRNAs (ARlncRNAs) (Table S3).

2.3. Construction of a Coexpression Network of the Autophagy-Related IncRNAs and Building a Sankey Diagram. Firstly, the clinicopathological data and ARlncRNAs were merged. Then, we constructed a mRNA-lncRNA interaction network of prognostic ARlncRNAs by applying Cytoscape software 3.8.0 [22]. A Sankey diagram was built to describe the relationship between the autophagy genes and lncRNAs by using the R software packages "ggalluvial" [23] and "ggplot2" [24].

2.4. Enrichment Analysis via DAVID Bioinformatics Resources. In this study, we conducted enrichment analyses via DAVID web server, a free server resource (https://david .ncifcrf.gov/summary.jsp) to elucidate the biological functions of those lncRNAs. 17 differentially expressed genes and 19 ARlncRNAs (Table S4) were taken to perform the analyses. Gene Ontology annotation and KEGG pathway were both involved.

2.5. Establishment of the ARlncRNA Signature and Performance Evaluation. First, the entire cohort obtained from the TCGA dataset was randomly divided into two cohorts: a training cohort (266 patients) and a validation cohort (264 patients). The training cohort was used to establish the Cox regression risk signature, and the validation cohort was then used to assess the performance of the 


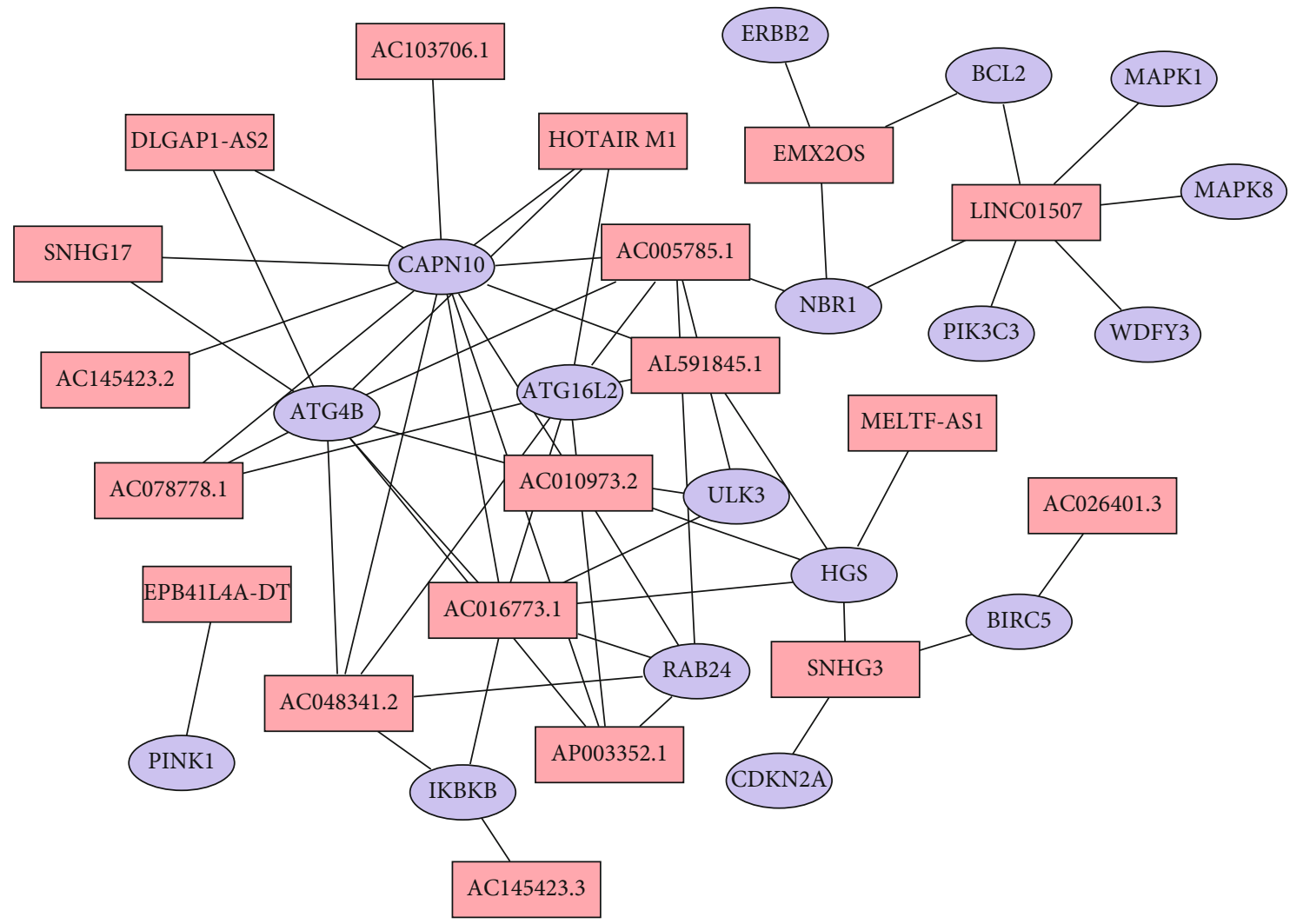

FIGURE 1: Network of prognostic lncRNAs with coexpressed autophagy genes in ccRCC. In the centric position, red nodes indicate lncRNAs, and blue nodes indicate autophagy genes. The coexpression network was visualized by CYTOSCAPE software 3.8.0.

signature. Then, univariate Cox regression and Kaplan-Meier (KM) analysis were used to identify ARlncRNAs with prognostic value. Seven $\operatorname{lncRNAs}$ considered significant with a $p$ value $<0.0001$ by both analyses were included in the resulting model. Next, multivariate stepwise regression Cox analysis was performed to establish a prognostic risk model. The risk score was calculated as the sum of multivariate Cox regression coefficient $(\beta)$-weighted expression levels of lncRNAs: Risk score $=\beta$ gene $(1) \times$ expression level of gene $(1$ )$+\beta$ gene $(2) \times$ expression level of gene $(2)+\cdots+\beta$ gene $(n) \times$ expression level of gene $(n)$. The training cohort was then separated into high-risk and low-risk groups based on the median risk score. We generated a receiver operating characteristic (ROC) curve to evaluate the predictive power of the risk score. The same analysis was adopted in the testing cohort to assess the performance of the signature. Additionally, we built a nomogram using the R package "rms" based on the results of multivariate Cox regression analysis to evaluate the prognosis of ccRCC. Lastly, the C-index and calibration curve were used to assess the performance value.

2.6. Independence Verification of the Risk Signature and Performance Evaluation. Univariate and multivariate Cox regression analyses were performed to investigate the independence of the risk signature as a predictive factor from the traditional clinical features (including age, sex, ISUP grade, and AJCC stage) in both the training and validation cohorts.
2.7. Statistical Analysis. In this study, all analyses were performed using $\mathrm{R}$ 4.0.2. A $p$ value $<0.05$ was considered statistically significant. The correlation matrix was constructed by $\mathrm{R}$ software based on Pearson correlation coefficients. The relationship between autophagy-related lncRNAs and overall survival was analyzed through the Kaplan-Meier curve, which was evaluated by the log-rank test. Time-dependent ROC curves were used to analyze the sensitivity and specificity of the prognostic prediction model. The nomogram was constructed with the regression coefficients based on the Cox analysis, and its performance was assessed by the cindex and calibration curve. Univariate and multivariate Cox regression analyses were performed to investigate the independence of the risk signature as a predictive factor, and Student's $t$-test was used to compare the clinicopathological features between different risk-score groups.

\section{Results}

3.1. Data Processing and Prognostic ARlncR Acquisition. We obtained transcriptome data and clinical data from the TCGA dataset. The basic clinical characteristics of the ccRCC patients in the TCGA database are shown in Table 1. Based on a total of 232 autophagy-related genes from the Human Autophagy Database (HADb, http:// autophagy.lu/clustering/index.html), we screened 813 autophagy-related lncRNAs. Nineteen ARlncRNAs closely connected to prognosis were identified in the training 


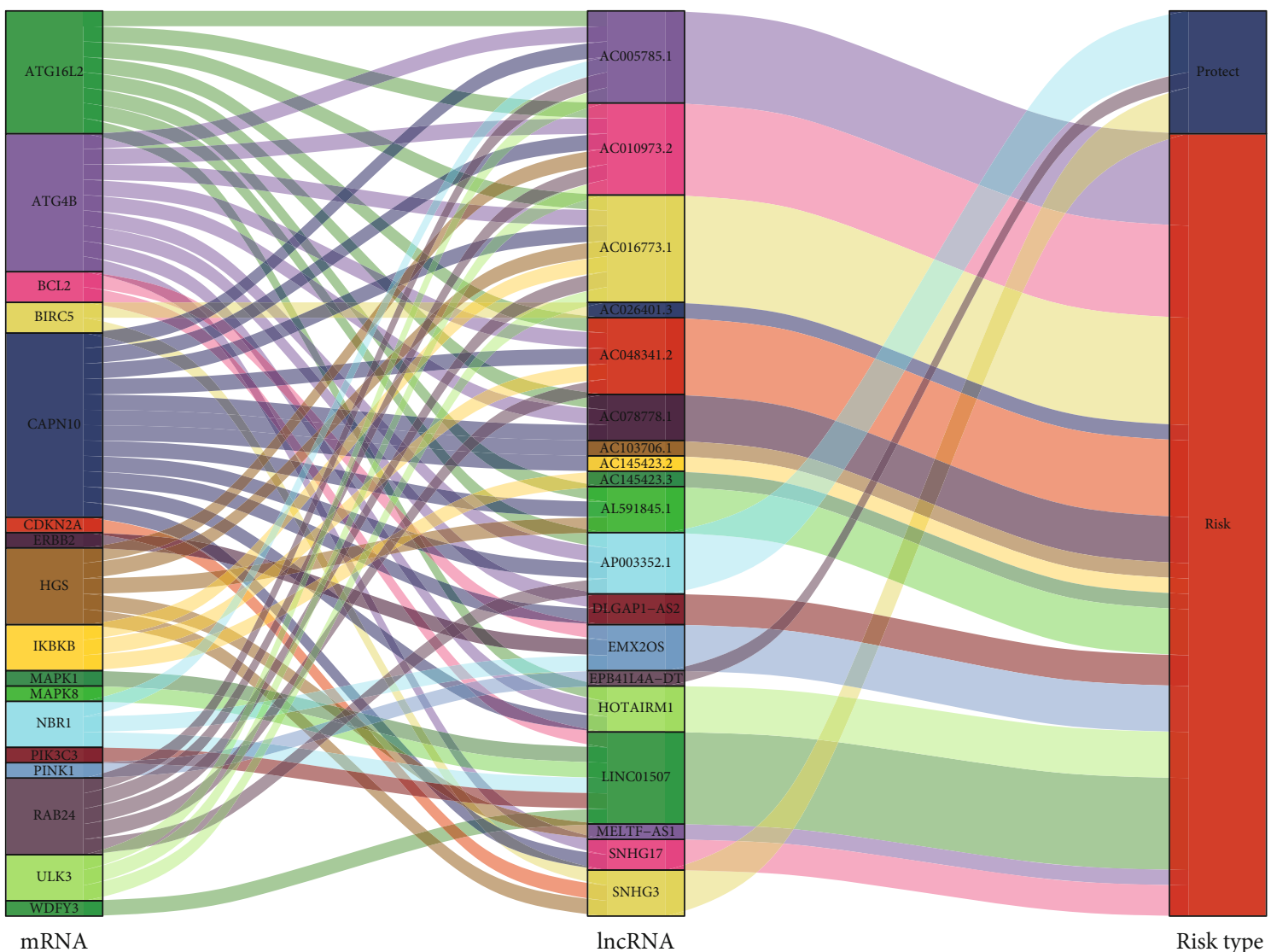

FIGURE 2: Correlation between ARlncRNAs and autophagy-related genes. Left bar: mRNA; middle bar: lncRNA; right bar: risk type. mRNA: messenger RNA; lncRNA: long noncoding RNA.

cohort through univariate Cox regression analysis ( $p$ value $<0.01$ ), and then, a network of prognostic 19 lncRNAs with coexpressed 17 autophagy genes in ccRCC and a Sankey diagram for visualization were built. The results are shown in Figures 1 and 2.

3.2. Enrichment Analyses. To further identify the Gene Ontology (GO) annotation and KEGG pathway in which the above lncRNAs were enriched, GO term and KEGG pathway enrichment analyses were performed via DAVID functional annotation tool. The visualization of results was achieved by "ggplot2" packages in R software. GO analysis showed that changes in the biological processes (BPs) of autophagy-related lncRNAs were enriched in mitophagy, peptidyl-threonine phosphorylation, positive regulation of translation, macroautophagy, and others (Table 2, Figure 3(a)). Changes in cell components (CCs) included the autophagosome, late endosome, and mitochondrion (Table 2, Figure 3(a)). Changes in molecular functions (MFs) were mainly enriched in kinase activity, protein kinase activity, protein serine or threonine activity, and others (Table 2, Figure 3(a)). KEGG pathway enrichment analysis indicated that those autophagy-related lncRNAs were involved in multiple tumor progressions and signaling pathways such as NOD-like receptor signaling pathway and mTOR signaling pathway (Table S5, Figure 3(b)).
3.3. Establishment of the Autophagy-Related $\operatorname{lncRNA}$ Signature for $c c R C C$. To improve our prognostic ability further, we employed stepwise multivariate Cox regression analysis to further evaluate the aforementioned ARlncRNAs. As a result, 7 lncRNAs were eventually pulled out to construct the signature. Herein, the risk score was assigned using a linear combination of the expression levels of the 7 identified lncRNAs weighted by their regression coefficients $(\beta)$ (Table 3, Figure 4). Then, the ccRCC patients were divided into high-risk and low-risk groups around the median risk score. As a result, the risk score distribution of the patients on the basis of the prognostic signature is shown in Figure 5(a). Survival status scatter plots for the patients based on the prognostic model are shown in Figure 5(b), indicating that patients in the high-risk group had a higher mortality than those in the low-risk group. A significant difference in overall survival between the two groups was seen (Figure 5(c), $p<0.0001$ ), and the AUCs at one, three, and five years were $0.754,0.791$, and 0.808 , respectively (Figures 5(d)).

3.4. Evaluation of the Prediction Performance of the Signature. Next, we assessed the predictive ability of the prognostic signature in the validation cohort to further evaluate its performance. The risk scores of the above 7 lncRNAs were recalculated for each patient in the validation cohort. As shown in (Figure 6(a)), the risk score distribution of the 


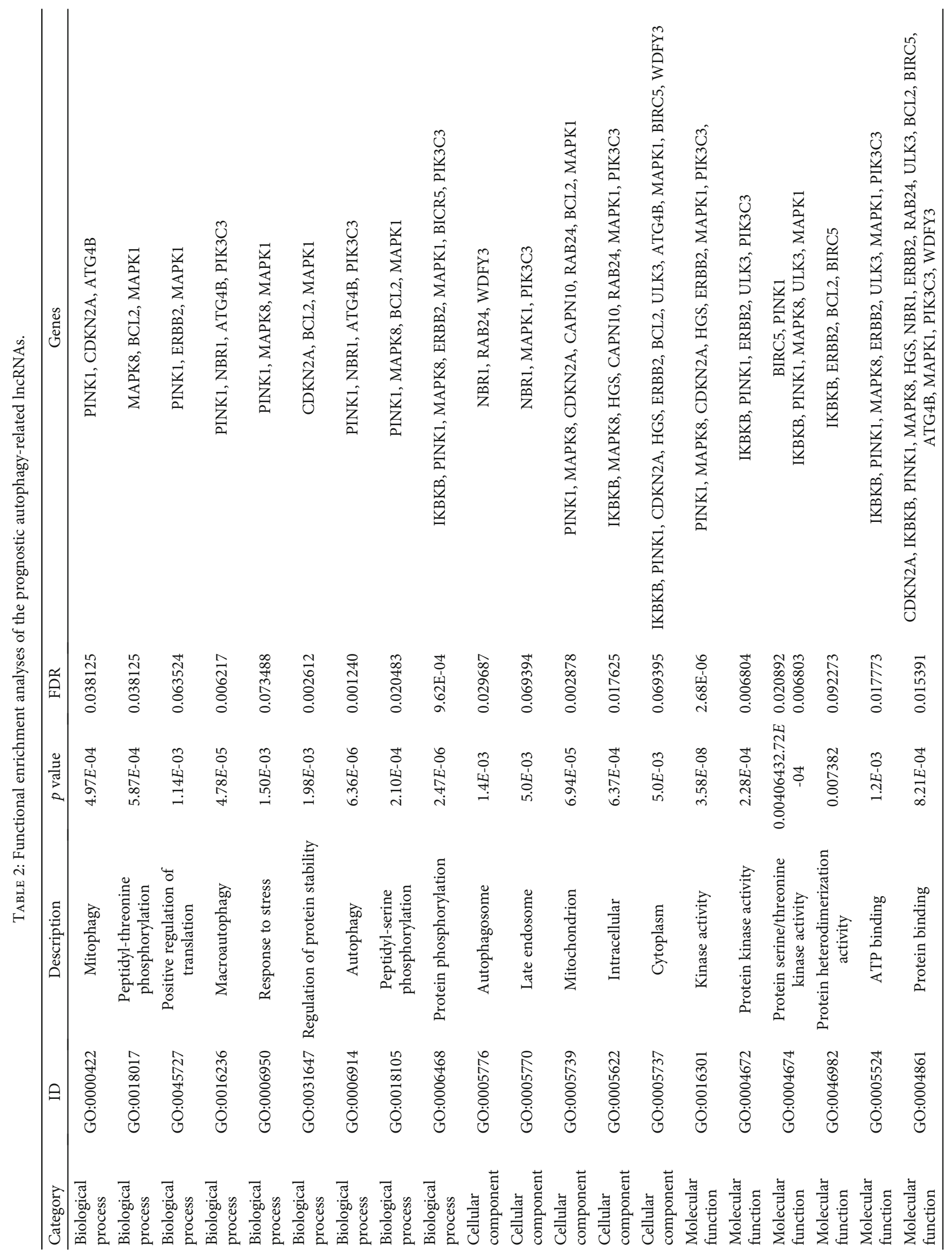




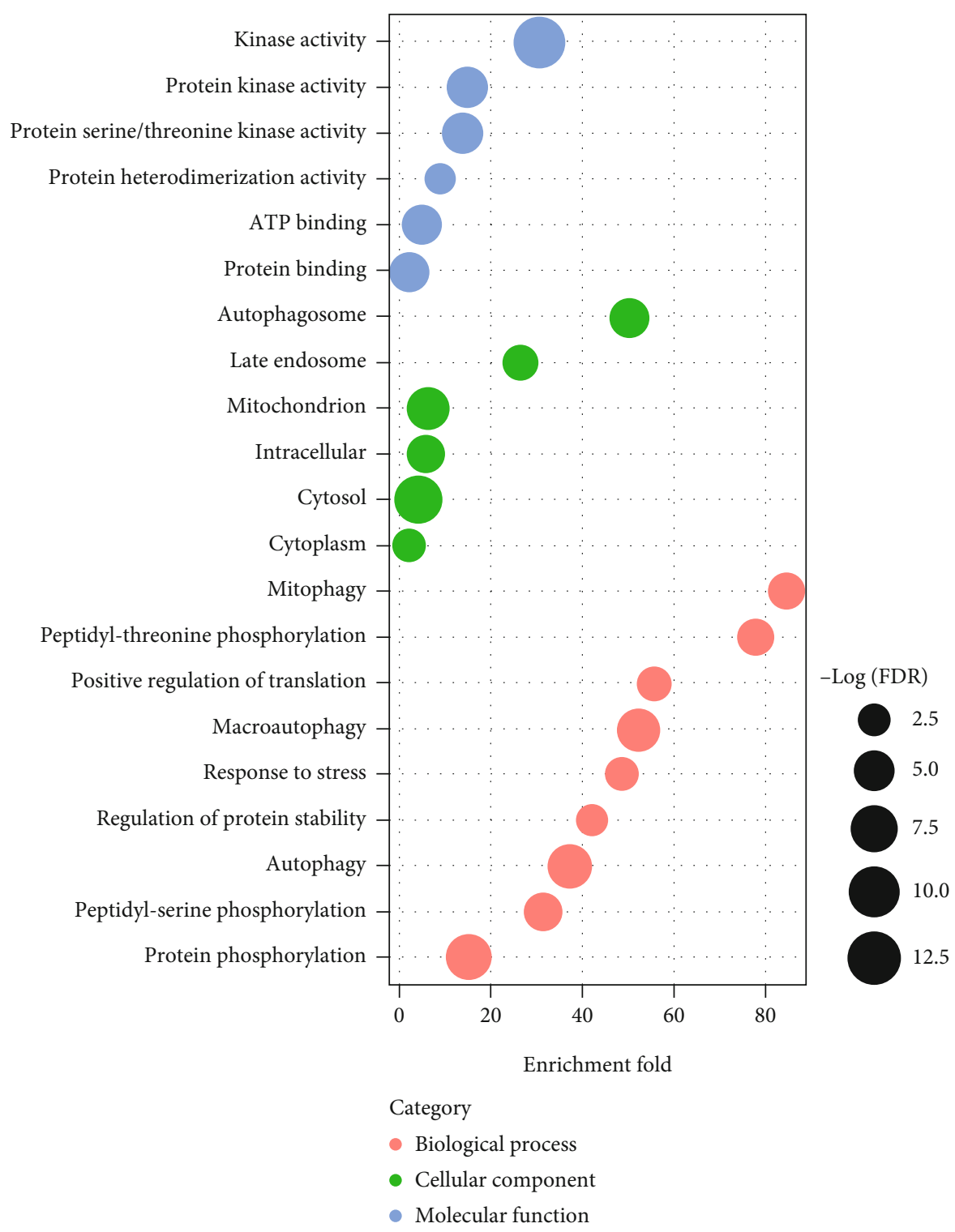

(a)

Figure 3: Continued. 


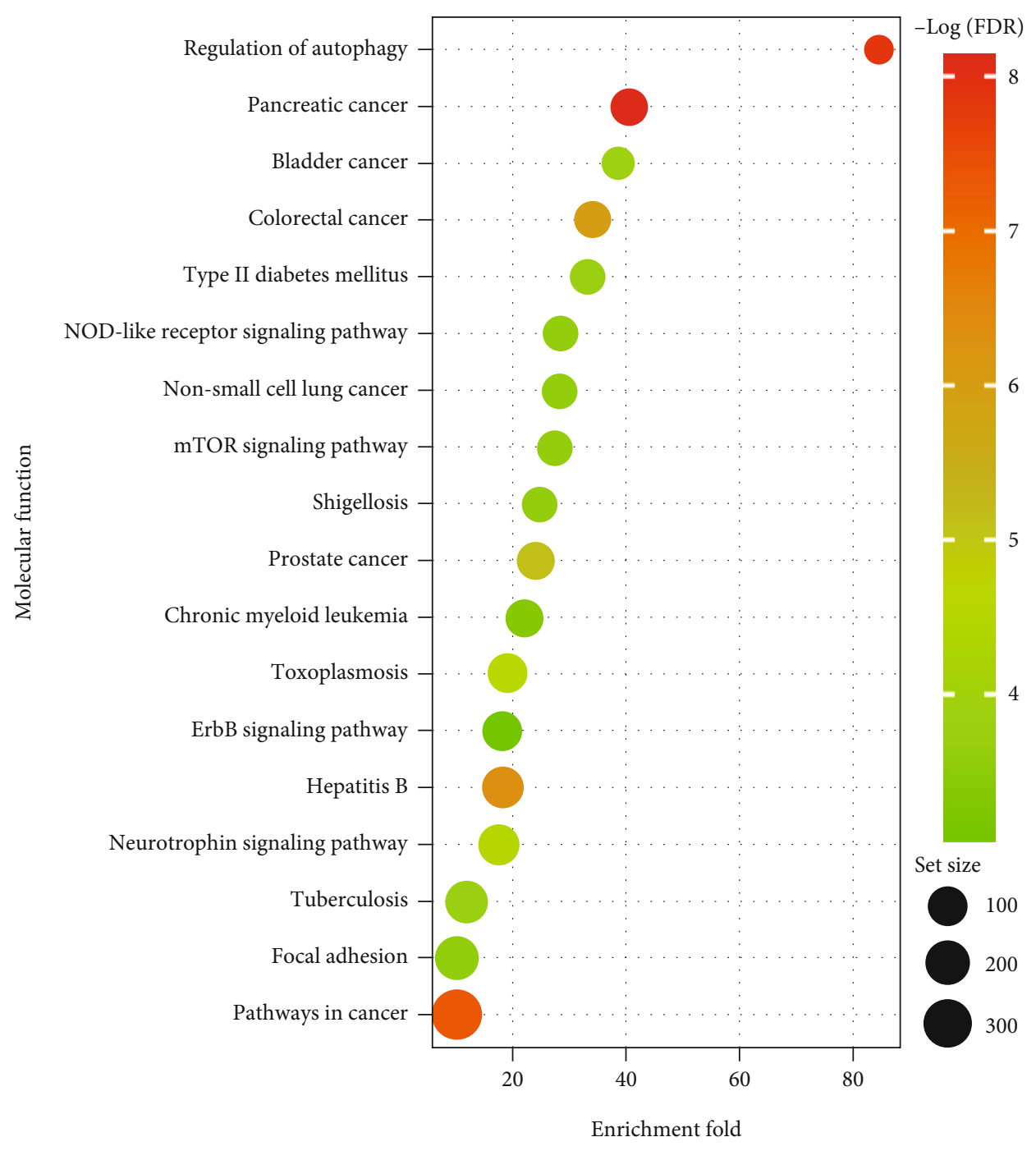

(b)

FIGURE 3: GO and KEGG pathway enrichment analysis. (a) GO enrichment analysis. Red nodes represent the changes in biological processes (BPs), green nodes represent the changes in cell components (CCs), blue nodes represent the changes in molecular functions (MFs). The $x$ -axis represents enrichment fold. (b) KEGG pathway enrichment analysis. The node color changes gradually from red to green in ascending order according to the $-\log (\mathrm{FDR})$ va. The size of each node represents the number of gene sets.

TABLE 3: Multivariate Cox regression analysis of prognostic autophagy-related genes.

\begin{tabular}{lcccrr}
\hline ID & Coef & HR & HR.95 L & HR.95 H & 0.970904 \\
SNHG3 & -0.647762 & 0.523215 & 0.281957 & 1.453059 & 4.759232 \\
SNHG17 & 0.966878 & 2.629724 & 1.094015 & 2.629247 & 0.040017 \\
MELTF-AS1 & 0.528276 & 1.696006 & 1.098048 & 2.456972 & 0.018193 \\
HOTAIRM1 & 0.496232 & 1.642520 & 0.104238 & 0.377789 & 0.015726 \\
EPB41L4A-DT & -1.617247 & 0.198444 & 0.168855 & 0.957802 & $8.51 \mathrm{E}-07$ \\
AP003352.1 & -0.910912 & 0.402157 & 0.923432 & 3.012484 & 0.039653 \\
AC145423.2 & 0.511553 & 1.667880 & & 0.089907 \\
\hline
\end{tabular}

patients based on the prognostic model presented similar results as in the training cohort. Survival status scatter plots for the patients on the basis prognostic model are presented in (Figure 6(b)). Survival differences were significant in the validation cohort (Figure 6(c)), $p<0.0001$ ). The AUCs at one, three, and five years were $0.686,0.673$, and 0.711 , respectively (Figures 6(d)). In addition, we constructed a nomogram to forecast the overall survival in the entire cohort 

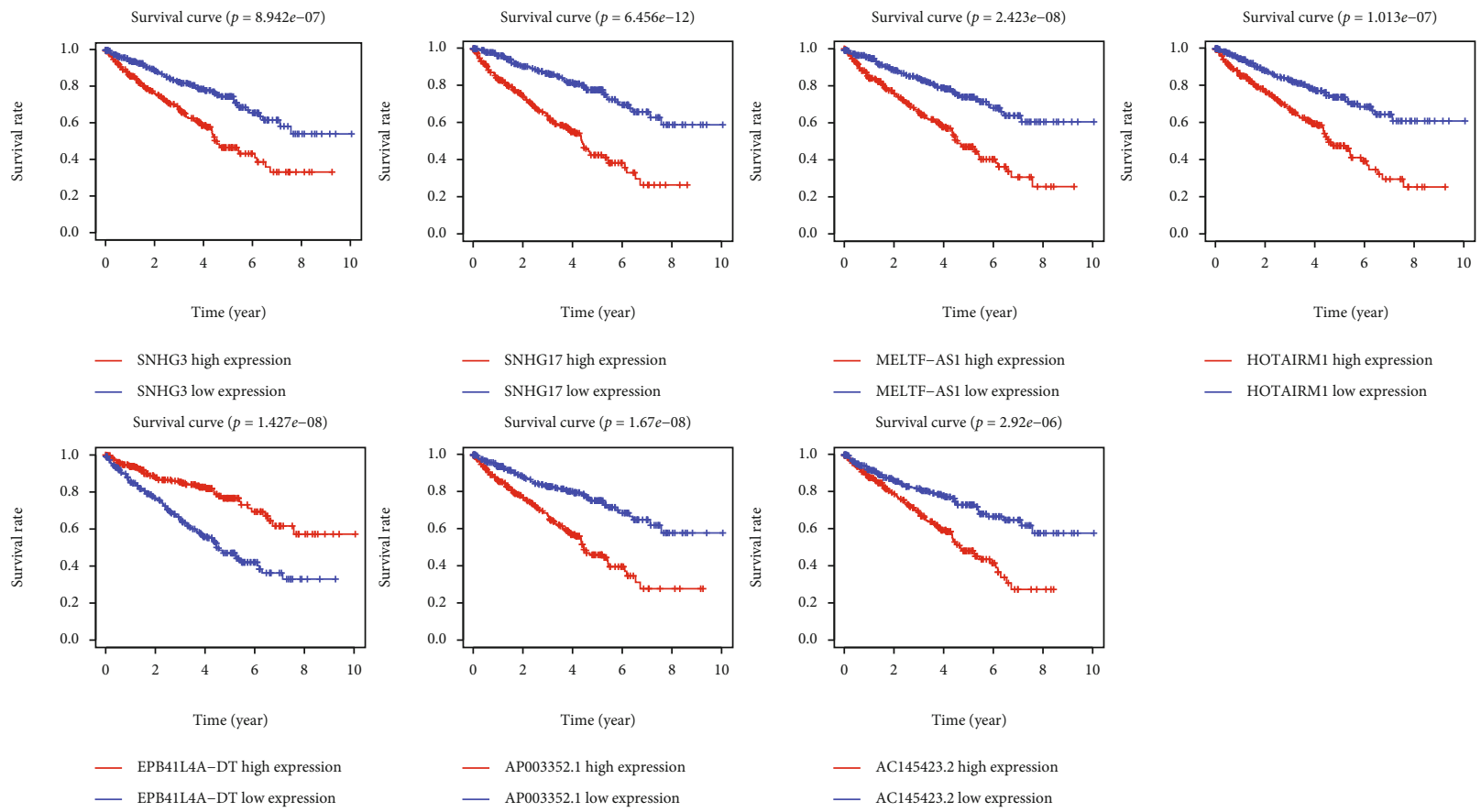

— HOTAIRM1 high expression

— HOTAIRM1 low expression

FIGURE 4: Kaplan-Meier survival curves for the 7 prognostic lncRNAs for ccRCC. The 7 autophagy-related lncRNAs were found to be of value in predicting prognosis in ccRCC patients.

with ccRCC according to the clinicopathological features and risk scores (Figure 7(a)), of which the C-index was 0.773 . The calibration curve for the nomogram suggested good performance. Thus, the nomogram proved to be of value in the prediction of the prognosis of ccRCC patients (Figures 7(b) and 7(c)).

\subsection{Independence Verification of the Signature as a Prognostic} Predictor. To further investigate whether the risk score could be predictive for ccRCC independent of conventional clinicopathological features (age, sex, ISUP grade, and AJCC state), univariate and multivariate Cox regression analyses were run. The risk score proved to be independent of the aforementioned clinical features in the entire cohort (Figure 8) (HR, 1.074, 95\% confidence interval: 1.036-1.113, $p<0.001$ ). The independence of the signature was further validated by stratified clinical features.

We conducted analyses to explore the value of the ARlncRNA signature in different clinicopathological subgroups, including age, sex, ISUP grade, AJCC stage, T stage, and $\mathrm{M}$ stage. Comparing the two results of each stratified feature revealed that the overall survival time of the high-risk group was shorter than that of the low-risk group in all clinicopathological subgroups (Figure 9). These results further indicated that the autophagy-related lncRNA signature can independently predict the prognosis of ccRCC patients.

\subsection{Exploring the Predictive Value of the Signature for Tumor} Progression. In order to get a deeper insight into the predictive value of the signature for ccRCC patients with regard to tumor progression, correlation analyses between the autophagy-related prognostic signature and clinicopathological features were performed. The risk score of stage III-IV
ccRCC was higher than that of stage I-II $(p=5.525 e-05$, Figure 10(a)). The risk score of G3-4 was higher than that of G1-2 ( $\mathrm{p}=4.247 \mathrm{e}-07$, Figure $10(\mathrm{~b}))$. The risk score of T3-4 was higher than that of T1-2 $(p=6.71 e-05$, Figure 10(c)). The risk score of M1 was higher than that of M0 $(p=0.015$, Figure $10(\mathrm{~d}))$, and the risk score of N1 was higher than that of N0 $(p=1.104 e-04$, Figure 10(e)). Taken together, these results indicated that as the risk score increased, the malignancy of ccRCC increased. Thus, the prognostic signature was of unique value for predicting the progression of ccRCC.

3.7. Relationships between the Prognostic ARlncRNAs and Clinicopathological Features. Subsequently, we investigated the connection between those ARlncRNAs and clinicopathological features. The purpose was to help develop a deeper understanding of the autophagy process, and the results shown in Table 4 revealed that each of those prognostic ARlncRNAs was significantly associated with clinicopathological features that are closely connected with tumor progression, including ISUP grade, AJCC stage, T stage, and $\mathrm{N}$ stage. SNHG17 and AP003352.1 were significantly associated with age. However, only EPB41L4A-DT was significantly associated with sex. Overall, these results indicated that the aforementioned ARlncRNAs can promote tumor progression.

\section{Discussion}

ccRCC is well known for its heterogeneity, exhibiting molecular diversity, morphological variability, and metabolic reprogramming. Late diagnosis without early warning signs and limited response to chemotherapy or radiotherapy are 


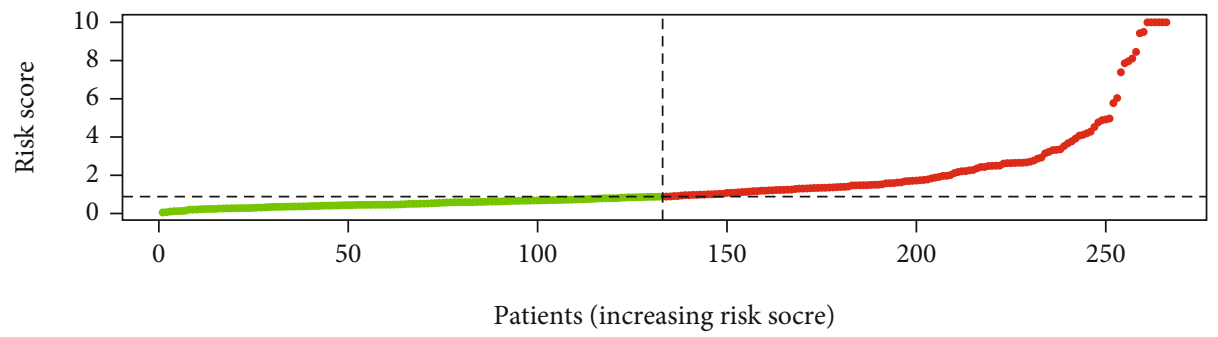

- High risk

- Low risk

(a)

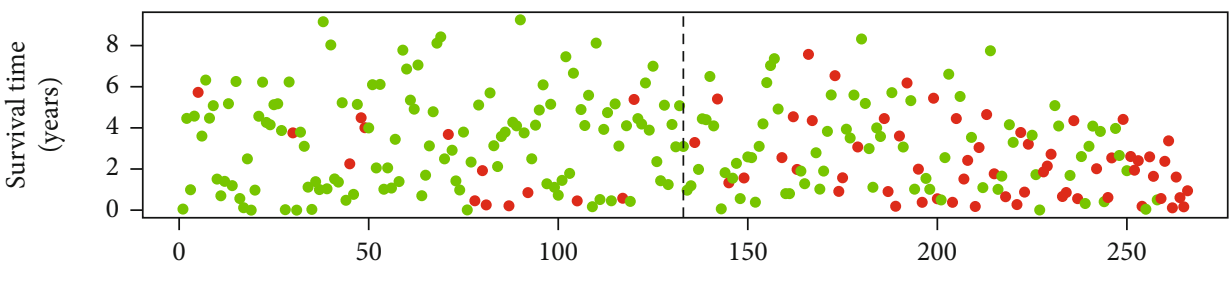

Patients (increasing risk socre)

- Dead

- Alive

(b)

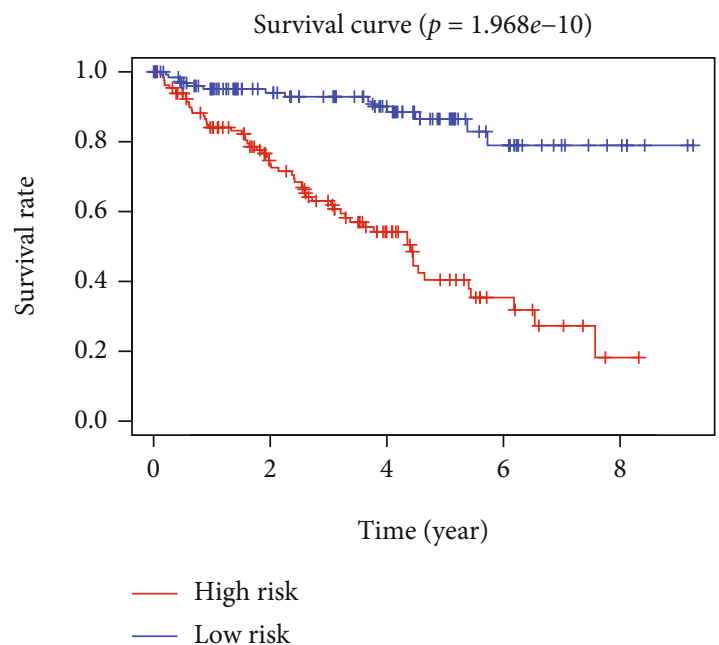

(c)

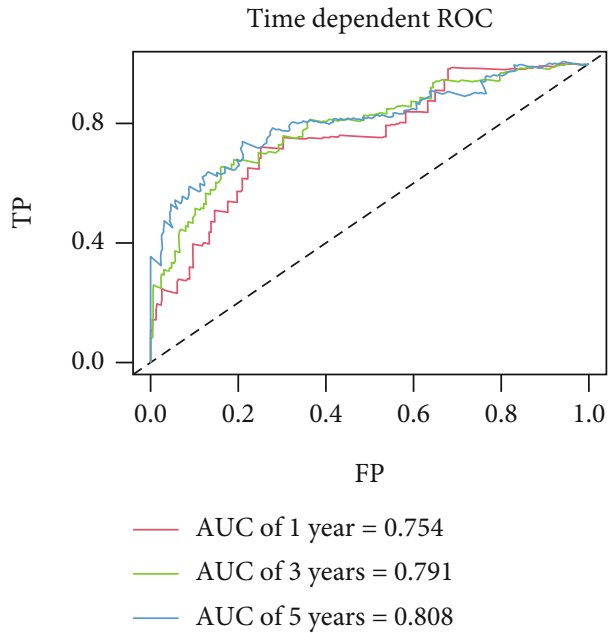

(d)

Figure 5: Prognostic analyses between the patients in the high-risk group and low-risk group in the training cohort. (a) Risk score distribution of patients from the prognostic signature. (b) Survival status scatter plots for patients in the prognostic signature (green dots: alive; red dots: death). (c) The Kaplan-Meier plot (high-risk vs. low-risk group) of the training cohort. (d) Time-dependent receiver operating characteristic curves assessed the predictive efficiency of the risk score for the training cohort. AUC: area under the curve; FP: false positive; TP: true positive.

the main culprits of poor prognosis [25]. Although novel biomarkers, especially autophagy-related genes and molecules, are emerging as predictive factors thanks to indepth cancer genetics and molecular biology discoveries $[26,27]$, the value of autophagy-related lncRNAs as prognostic indicators has not been addressed. Unlike previous studies that focused on the role of autophagy-related genes in tumorigenesis and progression [28-30], our study is aimed at improving prognostic prediction by finding autophagy-related lncRNAs associated with the poor prognosis of ccRCC through comprehensive bioinformatics analysis in TCGA databases.

We first identified $813 \operatorname{lncRNAs}$ on the basis of the IncRNA-autophagy gene coexpression network. By using a univariate Cox regression model, we identified 19 ARlncRNAs associated with the prognosis of ccRCC patients. Seven ARlncRNAs were further screened using multivariate Cox regression analysis, including SNHG3, SNHG17, MELTF- 


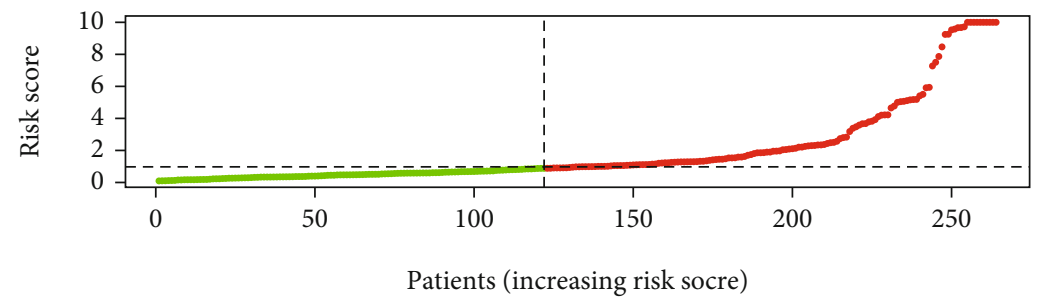

- High risk

- Low risk

(a)

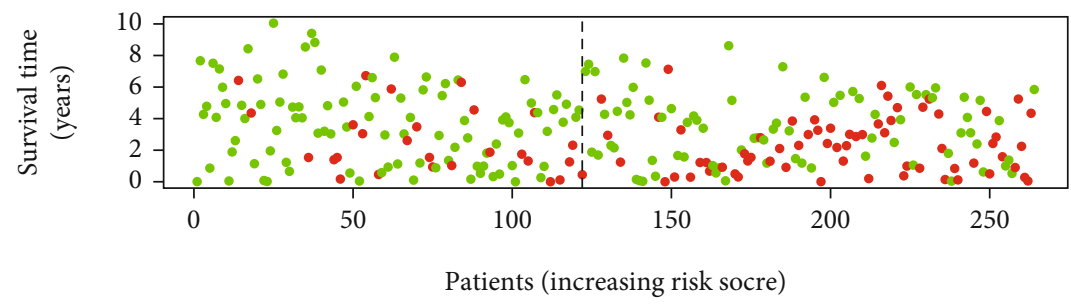

- Dead

- Alive

(b)

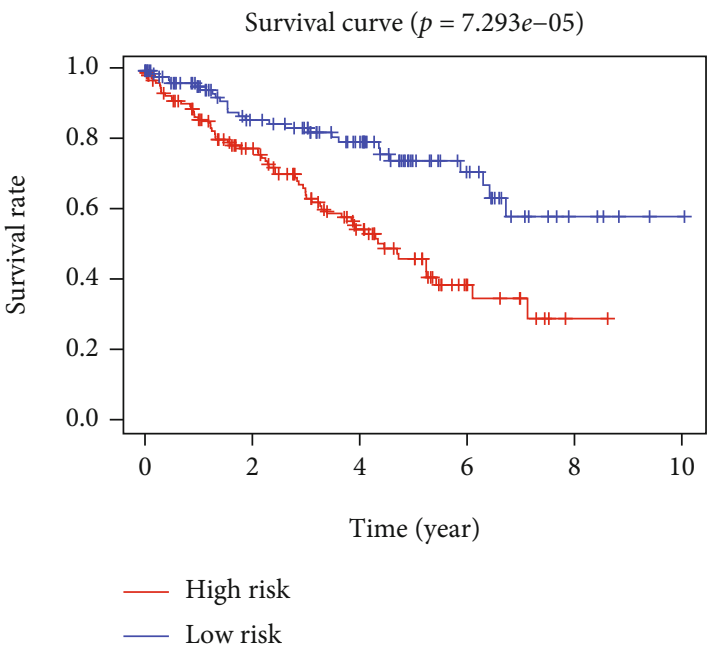

(c)

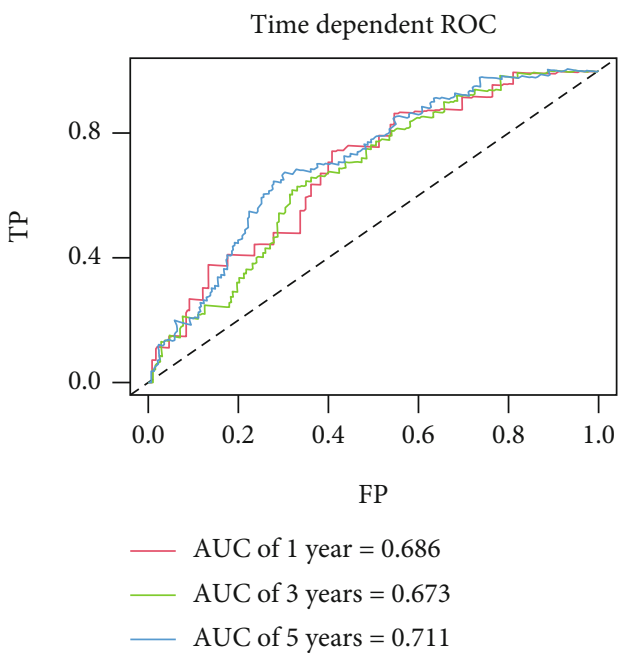

(d)

FIgURE 6: Prognostic analyses between the patients in the high-risk group and low-risk group in the validation cohort. (a) Risk score distribution of patients from the prognostic signature. (b) Survival status scatter plots for patients in the prognostic signature (green dots: alive; red dots: death). (c) The Kaplan-Meier plot (high-risk vs. low-risk group) of the training cohort. (d) Time-dependent receiver operating characteristic curves to assess the predictive efficiency of the risk score in the training cohort. AUC: area under the curve; FP: false positive; TP: true positive.

AS1, HOTAIRM1, EPB41L4A-DT, AP003352.1, and AC145423.2. GO analysis was conducted to discover the main biological characteristics of these ARlncRNAs. Next, we constructed a risk score-based prognostic signature that separated ccRCC patients into low-risk and high-risk groups. The OS time in the high-risk group was shorter than that in the low-risk group. The prediction performance was validated in the validation cohorts.

Furthermore, through univariate and multivariate Cox regression analyses, the risk score based on the signature was shown to be a prognostic factor for ccRCC independent of conventional clinicopathological features (age, sex, ISUP grade, and AJCC stage). Further evaluation demonstrated that the ARlncRNA signature can independently predict the progression of ccRCC, which means that the higher the risk score was, the worse the prognosis and the greater the degree of malignancy were. Finally, we established a nomogram based on the risk score of the signature, and the $\mathrm{C}$ index and calibration curve indicated that the predictive performance of the nomogram was good. Overall, these results indicate that our ARlncRNA signature can play an important role in predicting the prognosis of ccRCC patients. 


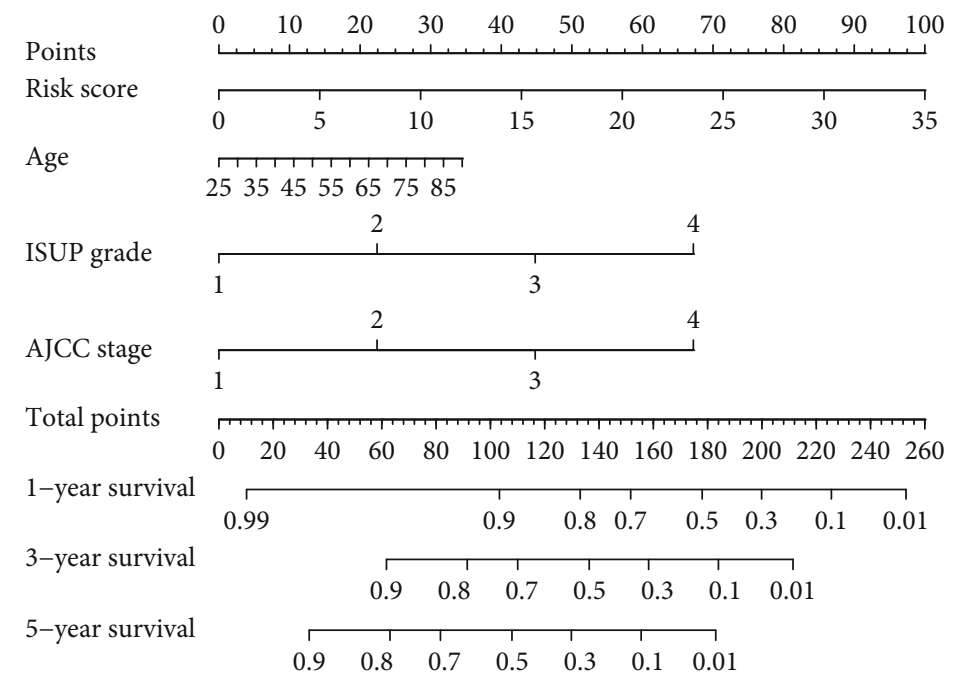

(a)

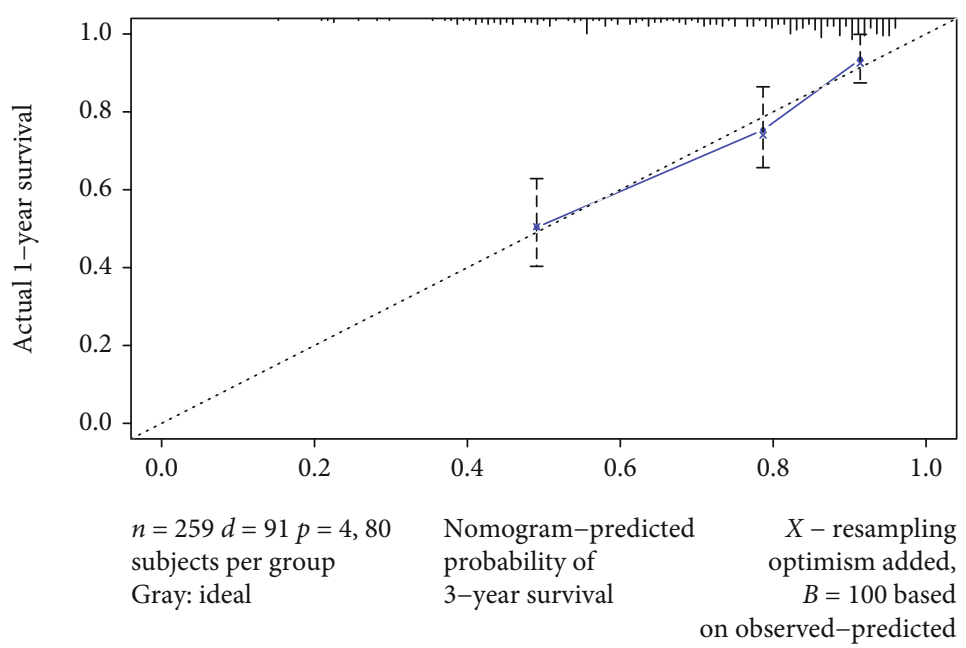

(b)

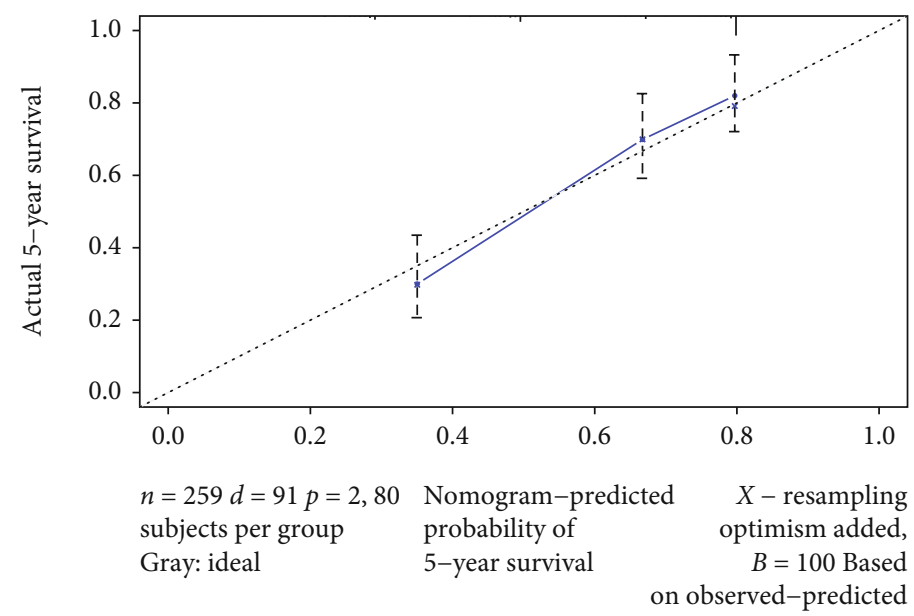

(c)

FIGURE 7: (a) Nomogram predicting 1-, 3-, and 5-year overall survival for patients with ccRCC. The calibration curve for predicting patient survival at (b) 3 years and (c) 5 years in the TCGA datasets. The nomogram-predicted probability of overall survival is plotted on the $x$-axis; actual overall survival is plotted on the $y$-axis. 

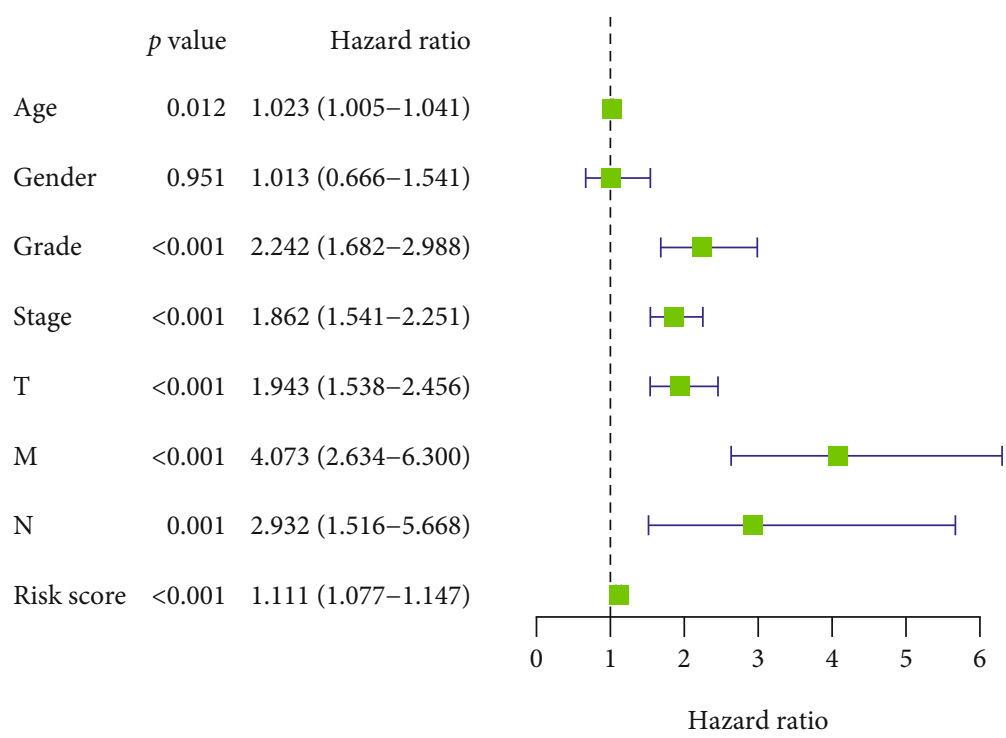

(a)

$\begin{array}{lrr} & p \text { value } & \text { Hazard ratio } \\ \text { Age } & <0.001 & 1.035(1.015-1.056) \\ \text { Gender } & 0.156 & 1.397(0.881-2.217) \\ \text { Grade } & 0.150 & 1.287(0.913-1.816) \\ \text { Stage } & 0.232 & 1.376(0.815-2.322) \\ \text { T } & 0.996 & 0.999(0.606-1.646) \\ \text { M } & 0.110 & 1.924(0.862-4.294) \\ \mathrm{N} & 0.329 & 1.424(0.700-2.895) \\ \text { Risk score } & <0.001 & 1.074(1.036-1.113)\end{array}$

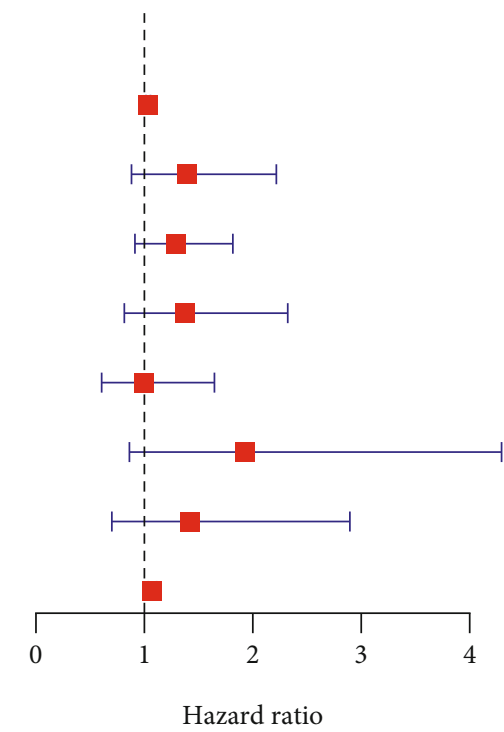

(b)

FIGURE 8: Univariate (a) and multivariate (b) Cox regression analyses for the TCGA cohort of ccRCC patients.

Autophagy is significantly connected to the prognosis of cancer; however, the complicated process and the numerous molecular interactions make autophagy play contradictory roles in cancer [31]. Long noncoding RNA small nucleolar RNA host gene 17 (SNHG17) was reported to be a critical regulator of tumorigenesis, and studies have reported its role in promoting tumor invasion and proliferation by activating the PI3K/AKT pathway [32, 33]. Jiang et al. [34] have reported that MELTF-AS1 can serve as a prognostic indicator and is associated with immunological processes. Our study further validates its prognostic value in association with the autophagy process, indicating that MELTF-AS can play distinct roles in multiple physio-pathological processes. HOTAIRM1 can promote glioblastoma progression [35], but in ccRCC, it is downregulated, serving as a suppressor of HIF1- dependent angiogenic pathways [36]. Thus, in vivo and in vitro experiments are required to illustrate the full effects of HOTAIRM1. Although we are the first to reveal the predictive value of EPB41L4A-DT, AP003352.1, and AC145423.2, their actual functions remain to be determined.

The common methods to detect lncRNA for clinical applications currently include microarray, lncRNA sequencing, and quantitative RT-PCR. Although the microarray can facilitate large-scale detections, its cost is relatively high [37]. LncRNA sequencing enables the detection to be more efficient and break the limit of traditional methods but leaving the library construction work tedious and expensive [38]. Quantitative RT-PCR is also a common method used to detect IncRNAs. It has been widely used because of its simplicity and low cost though it is still a low-throughput and 


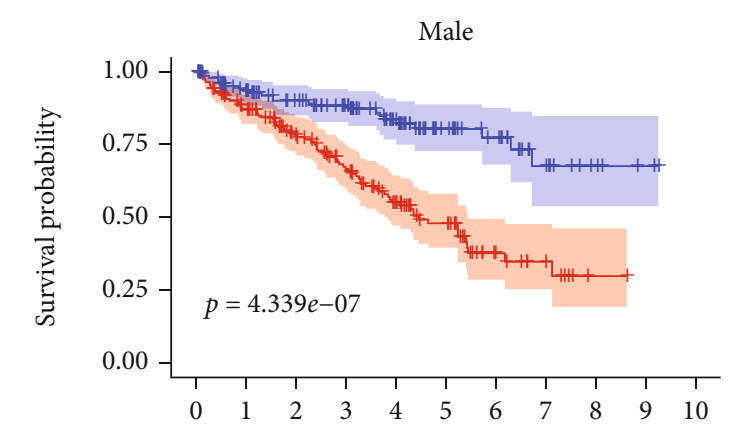

Time(years)

$\frac{\sqrt{3}}{21}$

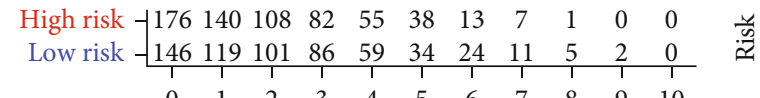

Time (years)

(a)

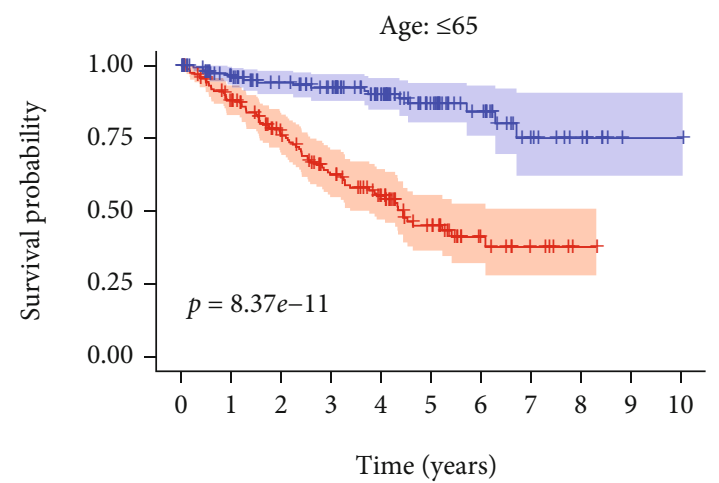

参 $\quad$ High risk $-166 \begin{array}{llllllllll}164 & 101 & 74 & 54 & 32 & 12 & 6 & 1 & 0 & 0\end{array}$

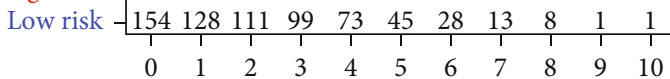

Time (years)

(c)

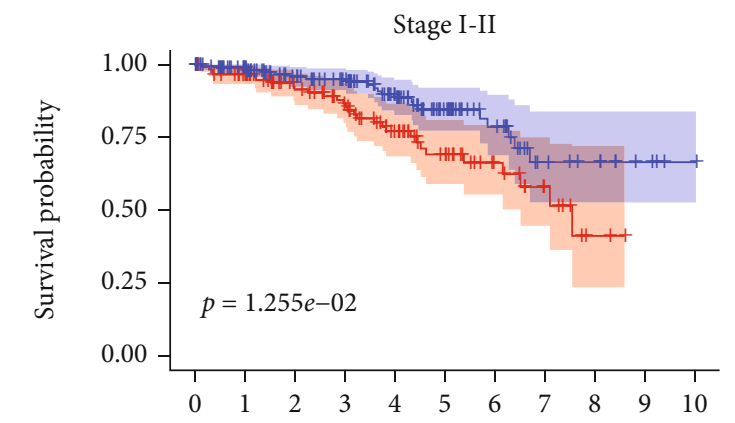

Time (years)

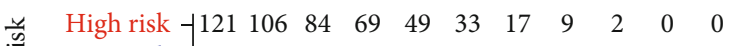
Low risk -\begin{tabular}{cccccccccccc}
165 & 141 & 119 & 105 & 76 & 46 & 27 & 12 & 9 & 4 & 1 \\
\hline 1 & 1 & 1 & 1 & 1 & 1 & 1 & 1 & 1 & 1 & 1 \\
0 & 1 & 2 & 3 & 4 & 5 & 6 & 7 & 8 & 9 & 10
\end{tabular}

Time (years)

(e)

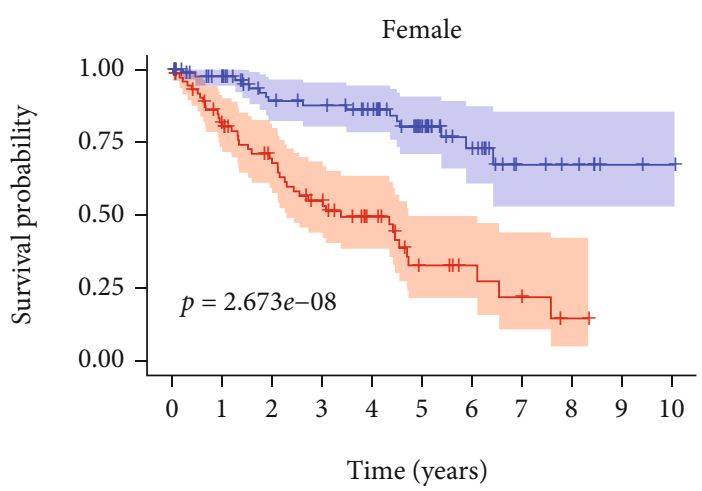

High risk $-\begin{array}{llllllllllll}75 & 55 & 42 & 31 & 21 & 10 & 6 & 3 & 1 & 0 & 0\end{array}$ Low risk -\begin{tabular}{rrrrrrrrrrr}
91 & 77 & 62 & 59 & 52 & 32 & 18 & 8 & 6 & 2 & 0 \\
\hline 0 & 1 & 1 & 1 & 1 & 1 & 1 & 1 & 1 & 1 & 1 \\
0 & 1 & 2 & 3 & 4 & 5 & 6 & 7 & 8 & 9 & 10
\end{tabular}

Time (years)

(b)

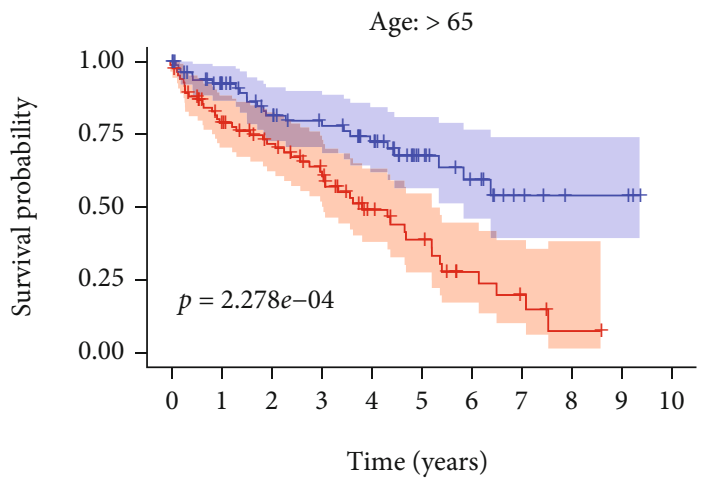

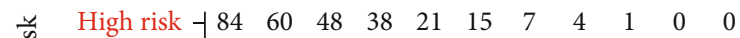

Low risk \begin{tabular}{rrrrrrrrrrr}
82 & 67 & 51 & 45 & 37 & 20 & 13 & 6 & 3 & 3 & 0 \\
\hline 0 & 1 & 1 & 1 & 1 & 1 & 1 & 1 & 1 & 1 & 1 \\
0 & 1 & 3 & 4 & 5 & 6 & 7 & 8 & 9 & 10
\end{tabular}

Time (years)

(d)

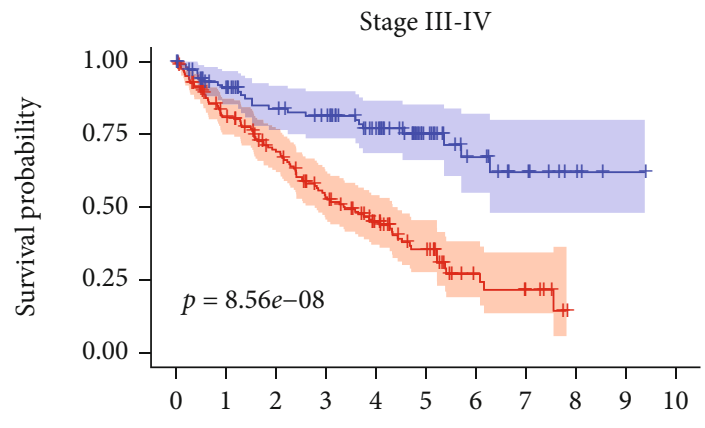

Time (years)

$\begin{array}{llllllllllll}\text { yै } & \text { High risk }-1163 & 122 & 97 & 69 & 46 & 29 & 10 & 6 & 0 & 0 & 0\end{array}$ Low risk -\begin{tabular}{ccccccccccc}
103 & 85 & 71 & 65 & 50 & 27 & 15 & 9 & 4 & 1 & 0 \\
\hline 1 & 1 & 1 & 1 & 1 & 1 & 1 & 1 & 1 & 1 & 1 \\
0 & 1 & 2 & 3 & 4 & 5 & 6 & 7 & 8 & 9 & 10
\end{tabular}

Time (years)

(f)

Figure 9: Continued. 
G1-2

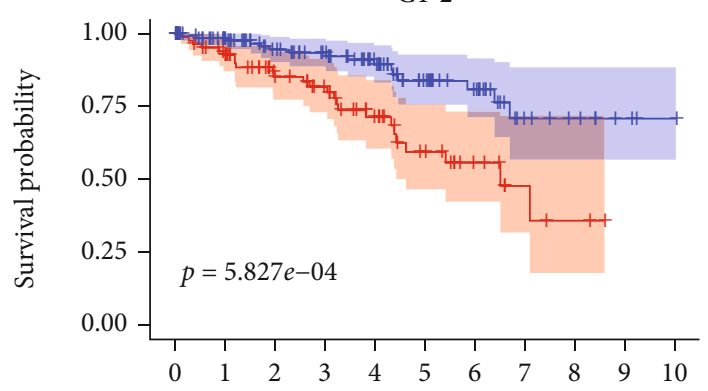

Time (years)

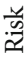

High risk $\dashv \begin{array}{llllllllllll}87 & 72 & 52 & 43 & 29 & 18 & 9 & 4 & 2 & 0 & 0\end{array}$ Low risk \begin{tabular}{rrrrrrrrrrr}
133 & 110 & 91 & 79 & 60 & 38 & 26 & 10 & 7 & 3 & 1 \\
\hline 0 & 1 & 2 & 3 & 4 & 5 & 6 & 7 & 8 & 9 & 10
\end{tabular} Time (years)

(g)

$\mathrm{T} 1-2$

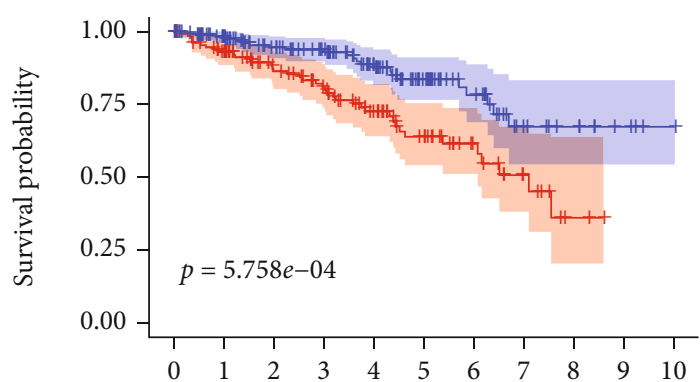

Time (years)

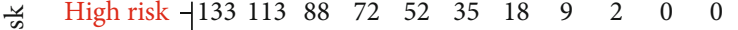
Low risk \begin{tabular}{|ccccccccccc}
170 & 145 & 122 & 107 & 78 & 48 & 29 & 13 & 9 & 4 & 1 \\
\hline 0 & 1 & 2 & 3 & 4 & 5 & 6 & 7 & 8 & 9 & 10
\end{tabular}

Time (years)

(i)
G3-4

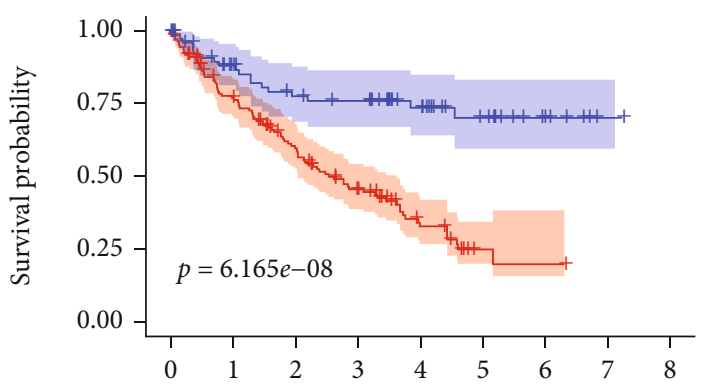

Time (years)

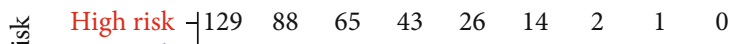
Low risk -\begin{tabular}{rrrrrrrrr}
71 & 54 & 43 & 39 & 34 & 19 & 14 & 7 & 2 \\
\hline 1 & 1 & 1 & $\mid$ & $\mid$ & $\mid$ & 1 & $\mid$ & 1 \\
0 & 1 & 2 & 3 & 4 & 5 & 6 & 7 & 8
\end{tabular} Time (years)

(h)

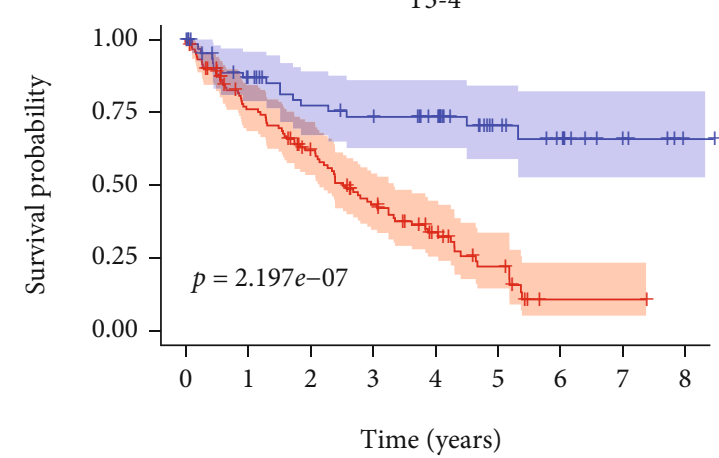

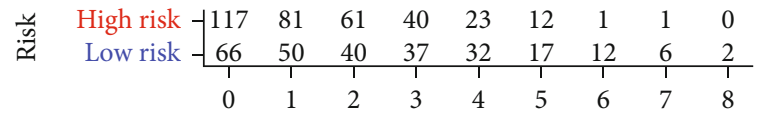
Time (years)

(j)

FIgure 9: Continued. 
M0

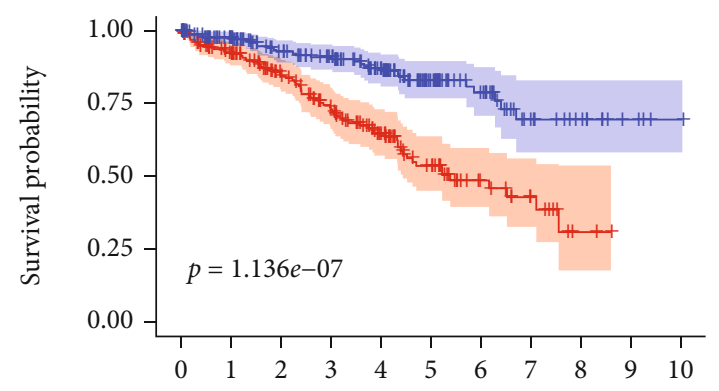

Time (years)

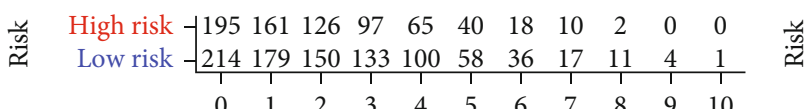

Time (years)

(k)
M1

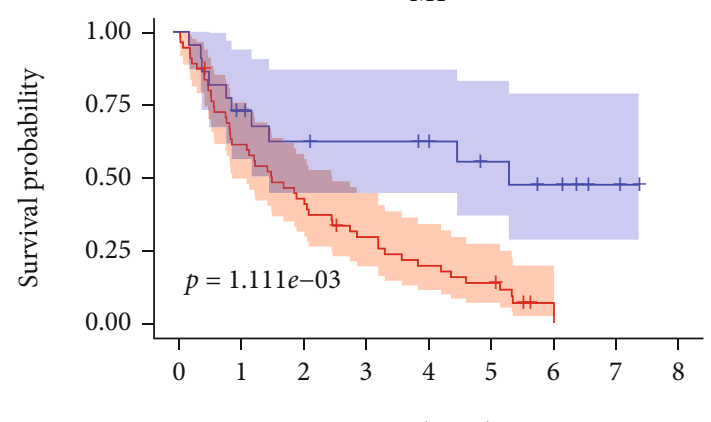

Time (years)

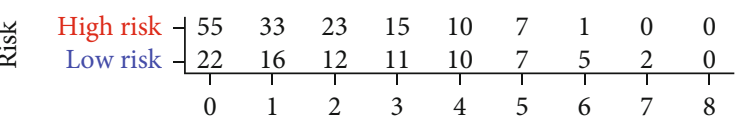

Time (years)
Risk

+ High risk

+ Low risk

(l)

FIgURe 9: Kaplan-Meier survival curves for the high-risk group and low-risk group stratified by clinicopathological features. (a, b) Sex. (c, d) Age. (e, f) Stage. (g, h) Grade. (i, j) T stage. $(k, 1)$ M stage. T: tumor size; M: metastasis.

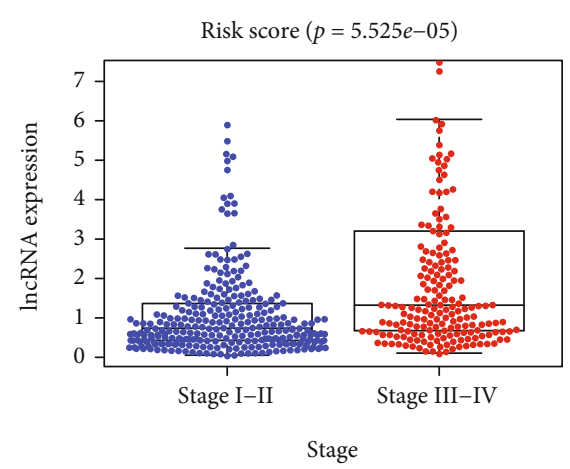

(a)

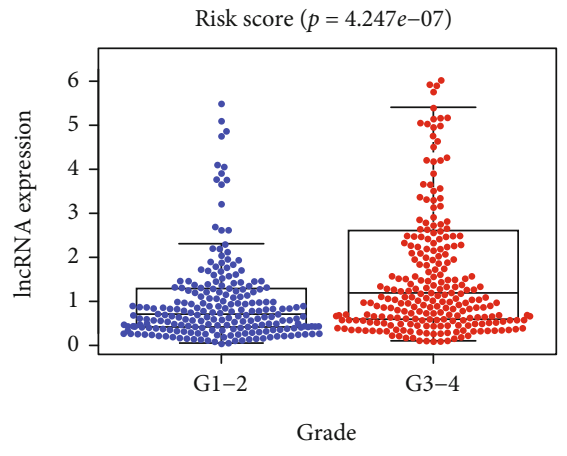

(b)

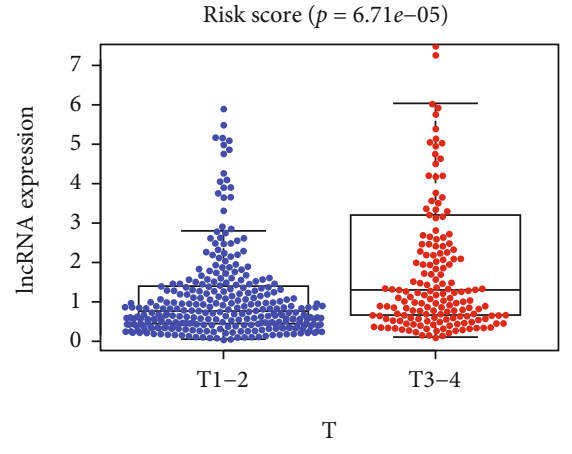

(c)

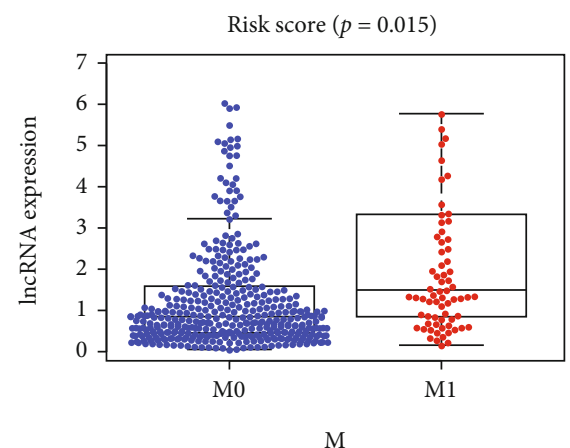

(d)

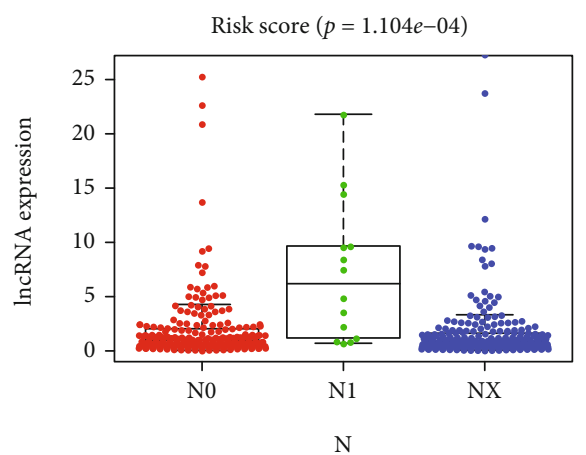

(e)

Figure 10: Evaluation of the predictive value of the signature for progression. (a) Stage. (b) Grade. (c) T stage. (d) M Stage. (e) N stage. T: tumor size; M: metastasis; N: lymph node metastasis. 
TABLE 4: Relationships between the prognostic ARGs and clinicopathological features.

\begin{tabular}{|c|c|c|c|c|c|c|c|c|c|c|c|c|}
\hline \multirow{2}{*}{$\operatorname{lncRNA}$} & & Age & \multicolumn{2}{|c|}{ Sex } & \multicolumn{2}{|c|}{ Grade } & \multicolumn{2}{|c|}{ Stage } & \multicolumn{2}{|c|}{ T stage } & \multicolumn{2}{|c|}{ M stage } \\
\hline & & $\leq 65>65$ & Female & Male & G1-2 & G3-4 & I-II & III-IV & $\mathrm{T} 1-2$ & T3-4 & M0 & M1 \\
\hline$N$ & & & 166 & 323 & 221 & 268 & 289 & 200 & 306 & 183 & 412 & 77 \\
\hline \multirow{2}{*}{ SNHG3 } & $t$ value & 1.81 & 0.1246 & & \multicolumn{2}{|c|}{3.660} & 4.565 & & 4.405 & & 2.791 & \\
\hline & $p$ value & 0.071 & 0.909 & & 0.0003 & & $<0.0001$ & & $<0.0001$ & & 0.0054 & \\
\hline \multirow{2}{*}{ SNHG17 } & $t$ value & 2.24 & 1.17 & & 3.152 & & 5.149 & & 4.959 & & 3.228 & \\
\hline & $p$ value & 0.0256 & 0.0017 & & 0.0017 & & $<0.0001$ & & $<0.0001$ & & 0.0013 & \\
\hline \multirow{2}{*}{ MELTF-AS1 } & $t$ value & 1.427 & 1.135 & & 4.319 & & 6.689 & & 6.185 & & 4.611 & \\
\hline & $p$ value & 0.1542 & 0.2571 & & $<0.0001$ & & $<0.0001$ & & $<0.0001$ & & $<0.0001$ & \\
\hline \multirow{2}{*}{ HOTAIRM1 } & $t$ value & 1.082 & 0.9008 & & 3.480 & & 4.407 & & 4.353 & & 2.503 & \\
\hline & $p$ value & 0.28 & 0.3682 & & 0.0005 & & $<0.0001$ & & $<0.0001$ & & 0.0126 & \\
\hline \multirow{2}{*}{ EPB41L4A-DT } & $t$ value & 0.6985 & 2.701 & & 5.920 & & 5.826 & & 7.712 & & 4.475 & \\
\hline & $p$ value & 0.4852 & 0.0072 & & $<0.0001$ & & $<0.0001$ & & $<0.0001$ & & $<0.0001$ & \\
\hline \multirow{2}{*}{ AP003352.1 } & $t$ value & 2.207 & 0.6670 & & 2.649 & & 4.72 & & 4.424 & & 2.814 & \\
\hline & $p$ value & 0.028 & 0.5051 & & 0.0083 & & $<0.0001$ & & $<0.0001$ & & 0.0051 & \\
\hline \multirow{2}{*}{ AC145423.2 } & $t$ value & 0.2175 & 0.2175 & & 3.921 & & 3.742 & & 2.836 & & 2.302 & \\
\hline & $p$ value & 0.8279 & 0.8279 & & 0.0001 & & 0.0002 & & 0.0048 & & 0.0218 & \\
\hline
\end{tabular}

low-specificity method [39]. Given those shortages, a comprehensive and personalized detection method of lncRNAs with further research achievements could be actually integrated into future precision medicine strategies.

Despite our novel findings, there are still limitations to this study. First, more basic experiments are required to clarify the mechanisms of action of ARlncRNAs in ccRCC tumor progression. Second, an external data set should be employed for validation, instead of our limited internal data set, to assess the consistency, reliability, and applicability of the autophagy-related signature.

\section{Conclusion}

In conclusion, we established an autophagy-related signature that independently predicted the prognosis of ccRCC patients. It can be used to guide individualized treatment regimens. Our study could help broaden the understanding of autophagy-related lncRNAs and narrow the gap between theoretical research and clinical practice, but the underlying mechanisms still urgently need to be understood to clarify the importance of our findings.

\section{Data Availability}

The data included in the current study were available in TCGA database (https://cancergenome.nih.gov/), the Human Autophagy Database (http://autophagy.lu/ clustering/index.html), and DAVID (https://david.ncifcrf .gov/summary.jsp).

\section{Ethical Approval}

Because the dataset in the current study was downloaded from TCGA and data acquiring and application complied with TCGA publication guidelines and data access policies, additional approval by an ethics committee and consent to participate were not needed.

\section{Conflicts of Interest}

The authors declared that they have no conflict of interest.

\section{Authors' Contributions}

Yundong Xuan and Shidong Zuo performed the data collection and the data analysis. Yundong Xuan and Kan Liu visualized and illustrated the results. Weihao Chen and Shidong Zuo conducted the statistic analyses. Yundong Xuan and Weihao Chen underwent the illustration of the results and carried out the initial manuscript writing. Baojun Wang and Xin Ma provided feedback on the manuscript and contributed to the study idea. All authors read and approved the final manuscript. Yundong Xuan and Weihao Chen contributed equally to this work.

\section{Supplementary Materials}

Table S1: 232 autophagy genes obtained from the Human Autophagy Database. Table S2: 210 autophagy genes expression matrix. Table S3: 813 autophagy-related lncRNAs. Table S4: 17 autophagy genes and 19 lncRNAs used to enrichment analyses. Table S5: results of enrichment via DAVID web serve. (Supplementary Materials)

\section{References}

[1] R. R. Kotecha, R. J. Motzer, and M. H. Voss, "Towards individualized therapy for metastatic renal cell carcinoma," Nature Reviews. Clinical Oncology, vol. 16, no. 10, pp. 621-633, 2019.

[2] D. J. Clark, S. M. Dhanasekaran, F. Petralia et al., "Integrated proteogenomic characterization of clear cell renal cell carcinoma," Cell, vol. 180, no. 1, p. 207, 2020. 
[3] M. B. Atkins and N. M. Tannir, "Current and emerging therapies for first-line treatment of metastatic clear cell renal cell carcinoma," Cancer Treatment Reviews, vol. 70, pp. 127-137, 2018.

[4] Y. Sato, T. Yoshizato, Y. Shiraishi et al., "Integrated molecular analysis of clear-cell renal cell carcinoma," Nature Genetics, vol. 45, no. 8, pp. 860-867, 2013.

[5] A. Rao, C. Wiggins, and R. C. Lauer, "Survival outcomes for advanced kidney cancer patients in the era of targeted therapies," Ann Transl Med, vol. 6, no. 9, p. 165, 2018.

[6] E. M. Posadas, S. Limvorasak, and R. A. Figlin, "Targeted therapies for renal cell carcinoma," Nature Reviews. Nephrology, vol. 13, no. 8, pp. 496-511, 2017.

[7] A. K. Lalani, B. A. McGregor, L. Albiges et al., "Systemic treatment of metastatic clear cell renal cell carcinoma in 2018: current paradigms, use of immunotherapy, and future directions," European Urology, vol. 75, no. 1, pp. 100-110, 2019.

[8] R. Amaravadi, A. C. Kimmelman, and E. White, "Recent insights into the function of autophagy in cancer," Genes \& Development, vol. 30, no. 17, pp. 1913-1930, 2016.

[9] G. Kroemer, G. Mariño, and B. Levine, "Autophagy and the integrated stress response," Molecular Cell, vol. 40, no. 2, pp. 280-293, 2010.

[10] J. M. Farrow, J. C. Yang, and C. P. Evans, "Autophagy as a modulator and target in prostate cancer," Nature Reviews. Urology, vol. 11, no. 9, pp. 508-516, 2014.

[11] E. White, J. M. Mehnert, and C. S. Chan, “Autophagy, metabolism, and cancer," Clinical Cancer Research, vol. 21, no. 22, pp. 5037-5046, 2015.

[12] H. M. Kang, K. H. Noh, T. K. Chang et al., "Ubiquitination of MAP1LC3B by pVHL is associated with autophagy and cell death in renal cell carcinoma," Cell Death \& Disease, vol. 10, no. 4, p. 279, 2019.

[13] T. R. Mercer and J. S. Mattick, "Structure and function of long noncoding RNAs in epigenetic regulation," Nature Structural \& Molecular Biology, vol. 20, no. 3, pp. 300-307, 2013.

[14] Y. Long, X. Wang, D. T. Youmans, and T. R. Cech, "How do lncRNAs regulate transcription?," Science Advances, vol. 3, no. 9, article eaao2110, 2017.

[15] F. Kopp and J. T. Mendell, "Functional classification and experimental dissection of long noncoding RNAs," Cell, vol. 172, no. 3, pp. 393-407, 2018.

[16] Z.-H. Chen, W.-T. Wang, W. Huang et al., "The IncRNA HOTAIRM1 regulates the degradation of PML-RARA oncoprotein and myeloid cell differentiation by enhancing the autophagy pathway," Cell Death and Differentiation, vol. 24, no. 2, pp. 212-224, 2017.

[17] L.-L. Wang, L. Zhang, and X.-F. Cui, “Downregulation of long noncoding RNA LINC01419 inhibits cell migration, invasion, and tumor growth and promotes autophagyviainactivation of the PI3K/Akt1/mTOR pathway in gastric cancer," Therapeutic advances in medical oncology, vol. 11, p. 175883591987465 , 2019.

[18] E. S. Martens-Uzunova, R. Böttcher, C. M. Croce, G. Jenster, T. Visakorpi, and G. A. Calin, "Long noncoding RNA in prostate, bladder, and kidney cancer," European Urology, vol. 65, no. 6, pp. 1140-1151, 2014.

[19] L. B. Frankel, M. Lubas, and A. H. Lund, "Emerging connections between RNA and autophagy," Autophagy, vol. 13, no. 1, pp. 3-23, 2017.
[20] Z. Wang, M. A. Jensen, and J. C. Zenklusen, "A practical guide to the cancer genome atlas (TCGA)," Methods in Molecular Biology, vol. 1418, pp. 111-141, 2016.

[21] M. E. Ritchie, B. Phipson, D. I. Wu et al., "limma powers differential expression analyses for RNA-sequencing and microarray studies," Nucleic acids research, vol. 43, no. 7 , p. e47, 2015.

[22] P. Shannon, A. Markiel, O. Ozier et al., "Cytoscape: a software environment for integrated models of biomolecular interaction networks," Genome Research, vol. 13, no. 11, pp. 24982504, 2003.

[23] J. Brunson, "Ggalluvial: layered grammar for alluvial plots," Journal of Open Source Software, vol. 5, no. 49, 2017.

[24] H. Wickham, ggplot2, Springer International Publishing, Cham, 2016.

[25] Cancer Genome Atlas Research Network, "Comprehensive molecular characterization of clear cell renal cell carcinoma," Nature, vol. 499, no. 7456, pp. 43-49, 2013.

[26] A. C. Kimmelman and E. White, "Autophagy and tumor metabolism," Cell Metabolism, vol. 25, no. 5, pp. 1037-1043, 2017.

[27] B. Levine and G. Kroemer, "Biological functions of autophagy genes: a disease perspective," Cell, vol. 176, no. 1-2, pp. 11-42, 2019.

[28] P. Chen, M. Cescon, and P. Bonaldo, "Autophagy-mediated regulation of macrophages and its applications for cancer," Autophagy, vol. 10, no. 2, pp. 192-200, 2014.

[29] G.-M. Jiang, Y. Tan, H. Wang et al., “The relationship between autophagy and the immune system and its applications for tumor immunotherapy," Molecular Cancer, vol. 18, no. 1, p. 17, 2019.

[30] A. Yang, N. V. Rajeshkumar, X. Wang et al., "Autophagy is critical for pancreatic tumor growth and progression in tumors with p53 alterations," Cancer Discovery, vol. 4, no. 8, pp. 905-913, 2014.

[31] B. Levine and G. Kroemer, "Autophagy in the pathogenesis of disease," Cell, vol. 132, no. 1, pp. 27-42, 2008.

[32] T. Xu, S. Yan, L. Jiang et al., "Gene amplification-driven long noncoding RNA SNHG17 regulates cell proliferation and migration in human non-small-cell lung cancer," Mol Ther Nucleic Acids, vol. 17, pp. 405-413, 2019.

[33] H. Gao, R. Liu, and X. Sun, "STAT3-induced upregulation of lncRNA SNHG17 predicts a poor prognosis of melanoma and promotes cell proliferation and metastasis through regulating PI3K-AKT pathway," European Review for Medical and Pharmacological Sciences, vol. 23, no. 18, pp. 8000-8010, 2019.

[34] Y. Jiang, X. Gou, Z. Wei et al., "Bioinformatics profiling integrating a three immune-related long non-coding RNA signature as a prognostic model for clear cell renal cell carcinoma," Cancer Cell International, vol. 20, no. 1, 2020.

[35] T. R. Spitzer, H. M. Lazarus, R. J. Creger, and N. A. Berger, "High-dose melphalan, misonidazole, and autologous bone marrow transplantation for the treatment of metastatic colorectal carcinoma. A phase I study," American Journal of Clinical Oncology, vol. 12, no. 2, pp. 145-151, 1989.

[36] M. J. Hamilton, M. Young, K. Jang et al., “_HOTAIRM1_ lncRNA is downregulated in clear cell renal cell carcinoma and inhibits the hypoxia pathway," Cancer Letters, vol. 472, pp. 50-58, 2020. 
[37] C. Zhang, W. Gao, S. Wen et al., "Potential key molecular correlations in laryngeal squamous cell carcinoma revealed by integrated analysis of mRNA, miRNA and lncRNA microarray profiles," Neoplasma, vol. 63, no. 6, pp. 888-900, 2016.

[38] X. Qian, J. Zhao, P. Y. Yeung, Q. C. Zhang, and C. K. Kwok, "Revealing lncRNA structures and interactions by sequencing-based approaches," Trends in Biochemical Sciences, vol. 44, no. 1, pp. 33-52, 2019.

[39] M. Kazimierczyk, M. K. Kasprowicz, M. E. Kasprzyk, and J. Wrzesinski, "Human long noncoding RNA interactome: detection, characterization and function," International journal of molecular sciences, vol. 21, no. 3, p. 1027, 2020. 